\title{
Safety of vaccination against brucellosis with the rough strain in pregnant cattle
}

\author{
Adriana Agostini Barbosa ${ }^{1,2}$ - Ana Cristina Silva Figueiredo ${ }^{1,2} \cdot$ Miller Pereira Palhao $^{1}$. \\ Joao Henrique Moreira Viana ${ }^{1,3} \cdot$ Carlos Antonio Carvalho Fernandes ${ }^{1,2}$
}

Received: 7 April 2017 / Accepted: 6 July 2017 /Published online: 15 August 2017

(C) Springer Science+Business Media B.V. 2017

\begin{abstract}
Brucellosis is an infectious and contagious disease that profoundly impacts public health. However, in many countries, disease prevention is restricted to the vaccination of calves, and there is no prophylactic strategy for pregnant heifers and cows. The aim of this study was to evaluate the safety of the rough strain vaccine against brucellosis in pregnant cattle. Crossbred cows $(N=96)$ at three gestational periods (early, mid, or late pregnancy) were randomly allocated into the vaccine treatment group or to the control group. We then compared the percentage of pregnancies reaching full term, live calves 60 days after delivery, and seropositive calves. There was no effect of vaccination in any of the gestational periods on the evaluation endpoints. In conclusion, vaccination against brucellosis with the rough strain is safe for pregnant cattle at all gestational periods.
\end{abstract}

Keywords Abortion · Brucella abortus · Calf survival . Pregnancy $\cdot$ Seroconversion $\cdot$ Vaccine

Carlos Antonio Carvalho Fernandes

carlos@biotran.com.br

1 Universidade José do Rosário Vellano, Rodovia MG 179, Km 0, Campus Universitário, Alfenas, MG 37130-000, Brazil

2 Biotran Biotecnologia e Treinamento em Reprodução Animal, Rua Tatuin 447, Alfenas, MG 37130-000, Brazil

3 Embrapa Recursos Genéticos e Biotecnologia, PqEB, Av. W5N, Brasília, DF 70770-917, Brazil

\section{Introduction}

Brucellosis is a highly transmissible infectious and contagious disease caused by the bacteria genus Brucella (Godfroid et al. 2011). It is a zoonosis with nearly worldwide distribution. Due to the lack of vaccines for humans, infection control relies on limiting the disease in animals. Cattle infection occurs mainly by the ingestion of water or food contaminated by uterine discharges, placental debris, fetuses after abortion, or parturition of animals with the disease, which may keep excreting the bacteria for up to 30 days. Thus, prevention of the disease in pregnant cattle must be a goal for the control of the transmission of the pathogen.

The vaccination of young ( 3 to 12 months) female calves with the S19 strain is common worldwide. However, vaccination of adult cattle with S19 is not recommended because it induces the production of specific antibodies that interfere in serological diagnosis and causes abortions in pregnant cattle (Godfroid et al. 2011). On the other hand, the rough strain vaccines such as RB51 do not induce the production of antibodies against lipopolysaccharide O (O-PS). Consequently, these vaccines do not interfere with conventional serologic diagnostic methods used to test adult cattle (Schurig et al. 2002). The RB51 vaccine has been successfully used in programs to control brucellosis outbreaks by mass vaccination of herds in countries such as the Azores (Martins et al. 2009) and Spain (Sanz et al. 2010). However, the effectiveness of the use of rough strain vaccines in adult cattle is controversial (Moriyón et al. 2004). Moreover, there are reports of abortion after vaccination with RB51 in endemic areas (Dougherty et al. 2013). Thus, the aim of this study was to evaluate the 
safety of the vaccination of pregnant cattle using a rough strain. Our hypothesis was that the vaccine would not increase the abortion rate, the number of newborn calf deaths or affect seroconversion to brucellosis regardless of when the vaccine was administered (early, mid, or late pregnancy).

\section{Materials and methods}

The study was performed in a farm located in the southern region of Minas Gerais State, Brazil. The regional climate is $\mathrm{Cw}$ according to Koepen's system (Peel et al. 2007). We examined crossbred Bos indicus $x$ Bos taurus cows and heifers $(N=96)$ with body weight ranging from 322 to $590 \mathrm{~kg}$ and body score conditions of 3.5 to 4.5 . All animals were vaccinated with the B19 strain when they were calves ( 3 to 8 month age) and were tested for brucellosis and tuberculosis 30 days before the experiment. When the experimental herd was formed, the animals were vaccinated against other diseases characterized by abortion, including leptospirosis, infectious bovine rhinotracheitis, and bovine viral diarrhea (CattleMaster Gold, Zoetis Brasil, Sao Paulo, Brazil). The animals received booster vaccines 30 and 60 days later. The pregnancies were confirmed by ultrasound (DP220, Mindray, Sao Paulo, Brazil) examinations of the uterus and fetus. The animals in each period of pregnancy (early pregnancy: 45 to 90 days [EP]; mid: 95 to 180 days [MP]; late pregnancy: 180 to 270 days [LP]) were randomly allocated into one of the following control or vaccinated groups: EP control $(82.2 \pm 10.7$ days; $N=16)$, EP vaccinated ( $84.0 \pm 11.3$ days; $N=16)$, MP control (163 \pm 23.9 days; $N=16$ ), MP vaccinated (177 \pm 27.1 days; $N=16)$, LP control ( $229 \pm 20.8$ days; $N=16$ ), and LP vaccinated $(236 \pm 22.4$ days; $N=16)$. The treated groups received a $2 \mathrm{~mL} \mathrm{SC}$ injection of a rough strain brucellosis vaccine (Brucelina Rebeccin batch 001/12, Vallée SA, Sao Paulo, Brazil) according to the manufacturer's instructions. The control groups received $2 \mathrm{~mL}$ of saline (placebo). During the experimental period, the cows and heifers were kept in an isolated area and raised under pasture (Brachiaria decumbens) conditions. The animals had ad libitum access to water and minerals. The pregnancies were monitored through term by visual inspection of clinical signs and eventual vaginal discharges. The data regarding parturitions and calves were recorded. When the calves were 60 days old, blood samples were collected from coccygeal vessels and analyzed for seroconversion to brucellosis using the acid plate antigen test (antigen bach 007/12, Tecpar, Curitiba, Brazil). The percentage of full term pregnancies, number of live calves at 60 days, and number of seropositive calves were compared among groups with Fisher's Exact Test (Ludbrook 2008) using a model that includes the effects of treatment, gestation period, and interactions. The data were analyzed with SAS software (SAS System for Information Delivery, v. 8.02, SAS Institute Inc., Cary, NC, USA). The results are presented as the mean \pm SD. Statistical significance was considered at $P<0.05$.

\section{Results and discussion}

The results are summarized in Table 1. There was no effect $(P>0.05)$ of vaccination on the proportion of pregnancies reaching term or in the percentage of live calves for any gestational period. The pregnancy losses were similar between the treated and control groups (8.3 vs. $10.4 \%$, respectively; $P>0.05)$ and are within the range reported for idiopathic pregnancy losses in dairy cattle (Diskin et al. 2011). There were no seropositive calves at 60 days born in either group.

Previous studies have addressed the occurrence of abortion in cattle after vaccination with RB51. However, these studies used a small number of experimental animals (Palmer et al. 1997; Uzal et al. 2000) or were performed in endemic areas (Dougherty et al. 2013). Moreover, the analysis of reproductive tissues indicates that the RB51 strain could infect bovine placentas, mammary glands, fetuses, and induce placentitis (Palmer et al. 1996). These results raise concerns regarding the safety of this vaccine. The novelty of the current study was our evaluation of RB51 vaccination effects in different periods of pregnancy (early, mid, and late pregnancy) using a relatively large number of animals in controlled conditions. We have demonstrated the vaccine has no effect on calf mortality
Table 1 Number and percentage of pregnancies to term and of live calves at 60 days in pregnant cattle vaccinated (treated group) or not vaccinated (control group) with a Brucella rough strain vaccine at different pregnancy periods

\begin{tabular}{llllll}
\hline Gestational period* & \multicolumn{2}{l}{ Pregnancies to term } & & \multicolumn{2}{l}{ Live calves } \\
\cline { 2 - 3 } \cline { 6 - 6 } & Control $N(\%)$ & Treated $N(\%)$ & & Control $N(\%)$ & Treated $N(\%)$ \\
\hline Early & $14 / 16(87.5)$ & $15 / 16(93.8)$ & & $12 / 14(85.8)$ & $14 / 15(93.3)$ \\
Mid & $14 / 16(87.5)$ & $13 / 16(81.3)$ & & $14 / 14(100.0)$ & $13 / 13(100.0)$ \\
Late & $15 / 16(93.8)$ & $16 / 16(100.0)$ & & $14 / 15(93.3)$ & $15 / 16(93.8)$ \\
Total & $43 / 48(89.6)$ & $44 / 48(91.7)$ & & $40 / 43(93.0)$ & $42 / 44(95.5)$ \\
\hline
\end{tabular}

For each endpoint (pregnancies to term; live calves), there was no difference between the control and treated groups $(P>0.05)$

${ }^{\text {a }}$ Early: 45 to 90 days; Mid: 95 a 180 days; Late: 180 to 270 days 
within the first 60 days. Additionally, the vaccine does not affect calf seroconversion to brucellosis. Calf survival postpartum is an important parameter in evaluating brucellosis vaccines from attenuated strains because it provides an indirect measurement of placental function. RB51 was previously reported to cause mild placentitis (Palmer et al. 1996). Thus, the vaccine could interfere with fetal development and consequently calf viability.

Cumulatively, our results suggest the rough strain vaccine against brucellosis is safe for pregnant cattle. Thus, it can be used in brucellosis control programs for the vaccination of female adult cattle regardless of pregnancy status.

Acknowledgements This research was supported by grants from Fundação de Amparo a Pesquisa de Minas Gerais (FAPEMIG) and the National Council for Scientific and Technological Development (CNPq, Brazil). The authors thank Vallée for supplying the vaccine batch used and Biotran LTD for supplying the animals.

Compliance with ethical standards All applicable international, national, and/or institutional guidelines for the care and use of animals were followed, and this study was approved by the Ethics in the Use of Animals Committee of the University José do Rosário Vellano (Protocol CEUA 22A/2012).

Conflict of interest The authors declare that they have no conflict of interest.

\section{References}

Diskin, M.G., Parr, M.H., Morris, D.G., 2011. Embryo death in cattle: an update. Reproduction Fertility and Development, 24, 244-251. doi: 10.1071/RD11914.

Dougherty, A.M.F., Cornish, T.E., O'Toole, D., Boerger-Fields, A.M., Henderson, O.L., Mills, K.W., 2013. Abortion and premature birth in cattle following vaccination with Brucella abortus strain RB51. Journal of Veterinary Diagnostic Investigation, 25, 630-635. doi: $10.1177 / 1040638713499570$.
Godfroid, J., Scholz, H.C., Barbier, T., Nicolas, C., Wattiau, P., Fretin, D., Whatmore, A.M., Cloeckaert, A., Blasco, J.M., Moriyon, I., Saegerman, C., Muma, J.B., Al Dahouk, S., Neubauer, H., Letesson, J.J., 2011. Brucellosis at the animal/ecosystem/human interface at the beginning of the 21st century. Preventive Veterinary Medicine, 102, 118-131. doi: 10.1016/j.prevetmed.2011.04.007.

Ludbrook J., 2008. Analysis of 2 x 2 tables of frequencies: matching test to experimental design. International Journal of Epidemiology, 37, 1430-1435. doi: 10.1093/ije/dyn162.

Martins, H., Garin-Bastuji, B., Lima, F., Flor, L., Pina Fonseca, A., Boinas, F., 2009. Eradication of bovine brucellosis in the Azores, Portugal-Outcome of a 5-year programme (2002-2007) based on test-and-slaughter and RB51 vaccination. Preventive Veterinary Medicine, 90, 80-89. doi: 10.1016/j.prevetmed.2009.04.002.

Moriyón, I., Grilló, M.J., Monreal, D., González, D., Marín, C., LópezGoñi, I., Mainar-Jaime, R.C., Moreno, E., Blasco, J.M., 2004. Rough vaccines in animal brucellosis: structural and genetic basis and present status. Veterinary Research, 35, 1-38.

Palmer, M.V., Cheville, N.F., Jensen, A.E., 1996. Experimental infection of pregnant cattle with the vaccine candidate Brucella abortus strain RB51: pathologic, bacteriologic, and serologic findings. Veterinary Pathology, 33, 682-691.

Palmer, M.V., Olsen, S.C., Cheville, N.F., 1997. Safety and immunogenicity of Brucella abortus strain RB51 vaccine in pregnant cattle. American Journal of Veterinary Research, 58, 472-477.

Peel M.C., Finlayson, B. L.,McMahon, T. A., 2007. Updated world map of the Köppen-Geiger climate classification. Hydrology and Earth System Science, 4,439-473.

Sanz, C., Sáez, J. L., Alvarez, J., Cortés, M., Pereira, G., Reyes, A., Rubio, F., Martin, J., Garcia, N., Domínguez, L., Hermoso-DeMendoza, M., Hermoso-De-Mendoza, J., 2010. Mass vaccination as a complementary tool in the control of a severe outbreak of bovine brucellosis due to Brucella abortus in Extremadura, Spain. Preventive Veterinary Medicine, 97, 119-125. doi: 10.1016/j. prevetmed.2010.08.003.

Schurig, G.G., Sriranganathan, N., Corbel, M.J., 2002. Brucellosis vaccines: past, present and future. Veterinary Microbiology, 90, 47996.

Uzal, F.A., Samartino, L., Shurig, G., Carrasco, A., Nielsen, K., Cabrera, R.F., Taddeo, H.R., 2000. Effect of vaccination with Brucella abortus strain RB51 on heifers and pregnant cattle. Veterinary Research Communications, 24, 143-151. 